\title{
Central tetrahydrobiopterin concentration in neurodevelopmental disorders
}

\author{
Richard E. Frye*
}

Department of Pediatrics, Division of Child and Adolescent Neurology and The Children's Learning Institute, University of Texas Health Science Center, Houston, TX, USA

\begin{abstract}
Tetrahydrobiopterin $\left(\mathrm{BH}_{4}\right)$ is a naturally occurring cofactor essential for critical metabolic pathways. Studies suggest that $\mathrm{BH}_{4}$ supplementation may ameliorate autism symptoms; the biological mechanism for such an effect is unknown. To help understand the relation between central $\mathrm{BH}_{4}$ concentration and systemic metabolism and to develop a biomarker of central $\mathrm{BH}_{4}$ concentration, the relationship between cerebrospinal fluid $\mathrm{BH}_{4}$ concentration and serum amino acids was studied. $\mathrm{BH}_{4}$ concentration was found to be distributed in two groups, a lower and higher $\mathrm{BH}_{4}$ concentration group. Two serum amino acids, citrulline and methionine, differentiated these groups, and the ratio of serum citrulline-to-methionine was found to correlate with the cerebrospinal fluid $\mathrm{BH}_{4}$ concentration $(r=-0.67, p<0.05)$. Both citrulline and methionine are substrates in inflammation and oxidative stress pathways - two pathways that utilize $\mathrm{BH}_{4}$ and are abnormally activated in autism. These data suggests that central $\mathrm{BH}_{4}$ concentration may be related to systemic inflammation and oxidative stress pathways.
\end{abstract}

\section{Keywords: tetrahydrobiopterin, autism, oxidative stress, inflammation, serum amino acids}

\section{INTRODUCTION}

Tetrahydrobiopterin, sapropterin, or $\mathrm{BH}_{4}$ is a naturally occurring essential cofactor for several critical metabolic pathways, including the production of monoamine neurotransmitters, the breakdown of phenylalanine, and the production of nitric oxide. Using $\mathrm{BH}_{4}$ as a cofactor, two aromatic amino acid hydroxylases, tyrosine-3hydroxylase and tryptophan-5-hydroxylase, catalyze the conversion of tyrosine and tryptophan to L-dopa and 5-hydroxytryptophan, respectively. These products are then further converted into dopamine and serotonin, respectively, and then further metabolized into norepinephrine and melatonin, respectively. $\mathrm{BH}_{4}$ is used as a cofactor with a third aromatic amino acid hydroxylase, phenylalanine-4-hydroxylase, to convert phenylalanine to tyrosine. Additionally, $\mathrm{BH}_{4}$ is essential in the production of nitric oxide, an important second messenger molecule used primarily for communication in vascular and neural tissues. In this reaction, nitric oxide synthase converts $\mathrm{L}$-arginine and oxygen to L-citrulline and nitric oxide.

$\mathrm{BH}_{4}$ is synthesized from guanosine- $5^{\prime}$-triphosphate, a purine nucleotide, by three enzymatic reactions (guanosine-5'triphosphate cyclohydrolase, 6-pyruvoyltetrahydropterin synthase, and sepiapterin reductase; Thony et al., 2000). A deficiency in $\mathrm{BH}_{4}$ production can result in two neurological disorders. Hyperphenylalaninemia, specifically phenylketonuria type IV, presents at birth with elevated phenylalanine in the blood and represents approximately $1-2 \%$ of phenylketonuria cases. This condition results from a reduction in phenylalanine-4-hydroxylase activity due to a deficiency in the necessary cofactor for this enzyme, $\mathrm{BH}_{4}$, and should be differentiated from classic phenylketonuria where phenylalanine-4-hydroxylase itself is defective. If phenylalanine levels are not controlled early in life in phenylketonuria, severe mental retardation will develop. $\mathrm{BH}_{4}$ deficiency can also result in dopamine-responsive dystonia, a neurological disorder that demonstrates a wide range of symptoms and age of presentations but invariably patients demonstrate dystonia with diurnal variation (Hyland et al., 1993).

Less well understood is the role of $\mathrm{BH}_{4}$ in neurodevelopmental disorders. Unconjugated 6R-5,6,6,8-tetrahyrobiopterin cerebrospinal fluid concentration in autistic children has been reported to be $42 \%$ lower than in neurotypical children. Further analysis of the autistic group found that only autistic children younger than 7 years old, but not older autistic children, demonstrated significantly lower 6R-5,6,6,8-tetrahyrobiopterin levels as compared to the control group. This younger group also demonstrated a reduction in total pterins and biopterin (Tani et al., 1994).

Several investigators have demonstrated a therapeutic effect of $\mathrm{BH}_{4}$ supplementation in children with autism. Over a 7-year period from 1984 to 1990, Japanese researchers demonstrated moderate or marked improvement in 41 to $64 \%$ in over 300 mildly to severely affected autistic children with $1-3 \mathrm{mg} / \mathrm{kg} /$ day of $\mathrm{BH}_{4}$ in five open-label and one double-blind placebo-controlled studies (Naruse et al., 1984, 1990a,b; Nagahata et al., 1990; Nakane et al., 1990; Takesada et al., 1992). In the double-blind placebo control study, Naruse et al. (1990a) demonstrated that the improvement in autism symptoms was limited to autistic children younger than 5 years of age. While these aforementioned studies treated children with autism without knowing cerebrospinal fluid $\mathrm{BH}_{4}$ concentrations, both Fernell et al. (1997) and Danfors et al. (2005) measured cerebrospinal fluid $\mathrm{BH}_{4}$ concentration and treated children with cerebrospinal fluid $\mathrm{BH}_{4}$ concentrations less than 12 and $30 \mathrm{nM} / \mathrm{L}$, respectively. Treating children with $3 \mathrm{mg} / \mathrm{kg} /$ day over a 12 -week period in an open-labeled manner, Fernell et al. (1997) found improvements in social responsiveness, communication, and cognitive abilities and demonstrated a decrease in the baseline elevation in $\mathrm{D}_{2}$ receptor binding found by positron emission tomography. In a 26-week double-blind, randomized, placebo-controlled trial Danfors et al. (2005) demonstrated 
an improvement in social interactions with a dose of $3 \mathrm{mg} / \mathrm{kg}$ of $\mathrm{BH}_{4}$ given twice a day. This improvement was found to be greater for autistic children with higher intelligence. Lastly, most recently, Frye et al. (2010) reported that five of eight (63\%) patients with autism and low pterin levels demonstrated improvement in social interactions and either verbal or non-verbal communication with treatment of $20 \mathrm{mg} / \mathrm{kg} /$ day of $\mathrm{BH}_{4}$ in an open-labeled manner.

Although studies have demonstrated that children with autism, as a group, have a reduced cerebrospinal fluid $\mathrm{BH}_{4}$ concentration and that some children with autism respond positively to $\mathrm{BH}_{4}$ supplementation, the biological mechanism for these findings has not been explained. It is clear from clinical studies that not all children with autism benefit from $\mathrm{BH}_{4}$ supplementation and that some children have side effects of $\mathrm{BH}_{4}$ supplementation, such as increased irritability and sleep disruption (Frye et al., 2010). Thus, understanding the biological mechanisms involved in $\mathrm{BH}_{4}$ supplementation is essential for determining which children may benefit from treatment with $\mathrm{BH}_{4}$ supplementation, understanding which underlying biological pathways are being modified with $\mathrm{BH}_{4}$ supplementation, and understanding which patients may be at risk for side effects of $\mathrm{BH}_{4}$ supplementation. To this end, this study examines whether biochemical markers of common metabolic pathways sampled from the blood are associated with cerebrospinal fluid $\mathrm{BH}_{4}$ concentration. Developing a biomarker of cerebrospinal fluid $\mathrm{BH}_{4}$ concentration would allow individuals with low central $\mathrm{BH}_{4}$ levels to be selected for supplementation without undergoing a lumbar puncture and would allow the effect of $\mathrm{BH}_{4}$ supplementation to be monitored during the treatment period in order to verify an adequate metabolic response to $\mathrm{BH}_{4}$ supplementation and optimize dosing of $\mathrm{BH}_{4}$ supplementation.

In order to consider what biochemical markers might respond to $\mathrm{BH}_{4}$ supplementation, the metabolic pathways that may benefit from $\mathrm{BH}_{4}$ supplementation in autism are considered. Here the focus is on children with autism since this is the population of individuals that has been found to have a positive response to $\mathrm{BH}_{4}$ (Frye et al., 2010). Examination of the metabolism characteristics unique to autism demonstrates that pathways that consume and recycle $\mathrm{BH}_{4}$ may be particularly dysfunctional in autism. For example, several lines of evidence suggest that children with autism manifest excessive inflammation and over activation of the immune system (Pardo et al., 2005; Dietert and Dietert, 2008; Castellani et al., 2009). Nitric oxide, a key mediator of inflammation and immune response, is produced by a $\mathrm{BH}_{4}$ dependent reaction. It is possible that a prolonged over activation of the immune system can result in excessive nitric oxide production and can, over time, deplete $\mathrm{BH}_{4}$. Children with autism might also have an underlying reduction in $\mathrm{BH}_{4}$ recycling for several reasons. Children with autism have been shown to have an impaired methylation capacity and markers of increased oxidative stress (James et al., 2004; Kern and Jones, 2006; Pardo and Eberhart, 2007; Deth et al., 2008). Folate is a key metabolite used to reduce oxidative stress. It is possible that a depletion of folate can result in reduced $\mathrm{BH}_{4}$ recycling through an over activation of the oxidative stress pathway. In addition, $\mathrm{BH}_{4}$ itself can act as an antioxidant, thus being depleted in the setting of high oxidative stress. Empirical evidence exists to support the notion that excess inflammation and oxidative stress can result in an acquired $\mathrm{BH}_{4}$ deficiency. Willoughby et al. (2009) demonstrated that $\mathrm{BH}_{4}$ levels were reduced below normal in three patients with subacute and chronic rabies infection. These investigators suggested that this acquired $\mathrm{BH}_{4}$ deficit was secondary to excess oxidation of $\mathrm{BH}_{4}$ and demonstrated significant neurological improvement in one of the two patients treated with $20 \mathrm{mg} / \mathrm{kg}$ of $\mathrm{BH}_{4}$.

Here it is suggested that prolonged excessive consumption and poor recycling of $\mathrm{BH}_{4}$ by non-central nervous system metabolic pathways deplete the available $\mathrm{BH}_{4}$ thereby preventing adequate $\mathrm{BH}_{4}$ levels in the central nervous system. To provide support for this notion, the relation between cerebrospinal fluid $\mathrm{BH}_{4}$ concentrations from a range of children with developmental and neurological disorders, and serum markers of oxidative stress and nitric oxide is examined. In this study serum amino acids are used as serum markers. It is hypothesized that these markers will be associated with cerebrospinal fluid $\mathrm{BH}_{4}$ concentration. Specifically it is hypothesized that a lower cerebrospinal fluid $\mathrm{BH}_{4}$ concentration will be associated with a reduction in methionine due to a reduced availability of folate and activation of the oxidative stress pathway (James et al., 2004), a reduction in arginine and an increase in citrulline due to an increase in the production of nitric oxide from inflammatory processes, and an increase in phenylalanine, tyrosine and tryptophan due to reduced aromatic amino acid hydroxylase activity.

\section{MATERIALS AND METHODS}

The goal of this study was to examine the association of amino acid biochemical markers with cerebrospinal fluid $\mathrm{BH}_{4}$ concentration. This required a population with a wide range of cerebrospinal fluid $\mathrm{BH}_{4}$ concentrations, and the patients reviewed in this study provided a wide range of cerebrospinal fluid $\mathrm{BH}_{4}$ concentrations. Including controls in the current study would not be appropriate due to ethical considerations. Specifically, the risk-benefit ratio of the lumbar puncture procedure is prohibitively high for healthy typically developing individuals who would not otherwise require such a procedure. A chart review found twenty patients who underwent a diagnostic lumbar puncture with measurement of cerebrospinal fluid $\mathrm{BH}_{4}$ concentration. All patients had a neurological diagnosis. Children with autism were diagnosed with developmental delays and encephalopathy and were distinguished from individuals with global developmental delay without autistic features.

Patients provided permission for review of their medical records through an Institutional Review Board approved protocol. Patient characteristics, including positive family history of neurologic or psychiatric disorder, findings from electroencephalogram and magnetic resonance imaging were abstracted from the charts. Cerebrospinal fluid was obtained through a lumbar puncture under general sedation to rule out metabolic and/or infectious disorders. The cerebrospinal fluid of all patients demonstrated a normal number of white and red blood cells, protein, glucose, 5-methyltetrahydrofolate and succinyladenosine concentrations. Cerebrospinal fluid was collected with standardized reagent tubes and frozen at $-80^{\circ} \mathrm{C}$ until $\mathrm{BH}_{4}$ analysis. $\mathrm{BH}_{4}$ content was measured by reversed-phase high performance liquid chromatography with electrochemical detection (Howells and Hyland, 1987). Eleven patients also had plasma amino acids measured within 4 months of the cerebrospinal fluid collection. Since plasma amino acids measured several times for most patients, the average values were calculated and used in further analysis. 
Cluster analysis using the Ward technique as implemented by the "CLUSTER" procedure of SAS was used to determine if there were groups with different cerebrospinal fluid $\mathrm{BH}_{4}$ concentrations, and if there were different groups, how many. Groups were analyzed with respect to differences in age, positive family history, spikes or slowing on electroencephalogram, abnormal magnetic resonance imaging and 5-hydroxyindoleacetic acid, homovanillic acid and 3-O-methyldopa concentrations. Defining these groups also allowed the assignment of prior probabilities for each group. These prior probabilities were important for examining the performance of the discriminant functions defined in the next step.

To determine which plasma amino acids were related to cerebrospinal fluid $\mathrm{BH}_{4}$ and could be used to predict the groups identified, a stepwise discriminant analysis as implemented by the "STEPDISC" procedure of SAS was used to select the plasma amino acids that, in combination, could differentiate the $\mathrm{BH}_{4}$ groups found. The specific plasma amino acids entered into the discriminant analysis included tyrosine, tryptophan, phenylalanine, citrul- line, arginine, and methionine. In a separate analysis, age was also added to the model since cerebrospinal fluid $\mathrm{BH}_{4}$ concentrations may be age dependent. Once the stepwise discriminant analysis selected the significant plasma amino acids, the selected variables were entered into the "DISCMIN" procedure in SAS, with and without prior probabilities, to determine the performance of the discriminant function.

\section{RESULTS}

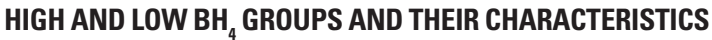

Cluster analysis was used to determine if there were distinct groups of patients with a common range of $\mathrm{BH}_{4}$ values. The cluster analysis identified two groups based on $\mathrm{BH}_{4}$ values: a cluster with 14 values with a cerebrospinal fluid $\mathrm{BH}_{4}$ concentration of $31 \mathrm{nM} / \mathrm{L}$ and below (mean $=20.14, \mathrm{SE}=0.160)$ and another cluster with 7 values with a cerebrospinal fluid $\mathrm{BH}_{4}$ concentration of $35 \mathrm{nM} / \mathrm{L}$ and above (mean $=39.43, \mathrm{SE}=1.24$; see Table 1). From these clusters we formed low and high cerebrospinal fluid $\mathrm{BH}_{4}$ groups. The low $\mathrm{BH}_{4}$

Table 1 | Participant characteristics.

\begin{tabular}{|c|c|c|c|c|c|c|c|c|}
\hline $\begin{array}{l}\text { Presenting } \\
\text { diagnosis }\end{array}$ & FHx & EEG & MRI & $\begin{array}{l}\text { Age }(\mathrm{m}) / \\
\text { gender }\end{array}$ & $\begin{array}{l}\text { BH4 } \\
\text { (nM/L) }\end{array}$ & $\begin{array}{l}\text { 5-HIAA } \\
\text { (nM/L) }\end{array}$ & $\begin{array}{l}\text { HVA } \\
\text { (nM/L) }\end{array}$ & $\begin{array}{l}\text { 3OMD } \\
\text { (nM/L) }\end{array}$ \\
\hline GDD & $\mathrm{MM}$ & $\mathrm{NL}$ & $\mathrm{NL}$ & $15 / F$ & 12 & 332 & 669 & 24 \\
\hline GDD & ED, MR & ND & $\mathrm{NL}$ & $14 / \mathrm{M}$ & 12 & 301 & 479 & 91 \\
\hline Chorea & NS & $\mathrm{NL}$ & $\begin{array}{l}\text { Punctate } \\
\text { lesions }\end{array}$ & $204 / F$ & 13 & 129 & 189 & 12 \\
\hline Autism & Mom Sz & $\mathrm{NL}$ & $\begin{array}{l}\text { Posterior } \\
\text { fossa cyst }\end{array}$ & $72 / \mathrm{M}$ & 17 & 89 & 342 & 18 \\
\hline Autism & NS & $\mathrm{NL}$ & $\mathrm{NL}$ & $48 / F$ & 19 & 191 & 636 & 38 \\
\hline GDD & ED & Slow & PVL & $67 / \mathrm{M}$ & 19 & 281 & 573 & 16 \\
\hline Autism & NS & $\mathrm{NL}$ & $\mathrm{NL}$ & 30/M & 19 & 177 & 558 & 83 \\
\hline $\begin{array}{l}\text { Refractory } \\
\text { seizures }\end{array}$ & Sz, Psych & $\mathrm{NL}$ & Volume loss & 168/M & 20 & 179 & 374 & 18 \\
\hline Autism & NS & $\mathrm{NL}$ & DWMA & $65 / M$ & 21 & 230 & 414 & 24 \\
\hline GDD & NS & Slow & Volume loss & $42 / \mathrm{M}$ & 21 & 226 & 720 & 35 \\
\hline GDD & NS & $\mathrm{NL}$ & $P V L$ & $63 / F$ & 23 & 201 & 318 & 12 \\
\hline Dystonia & NS & ND & $\mathrm{NL}$ & $52 / \mathrm{M}$ & 27 & 363 & 359 & 61 \\
\hline & & & & & 28 & 177 & 234 & 25 \\
\hline Autism & $S z, A D$ & $\begin{array}{l}\text { Multifocal } \\
\text { spikes }\end{array}$ & PVL & $32 / \mathrm{M}$ & 31 & 195 & 599 & 26 \\
\hline SD & $\mathrm{Sz}$ & Focal spikes & $\mathrm{NL}$ & $38 / F$ & 35 & 226 & 720 & 35 \\
\hline Autism & PDD & $\mathrm{NL}$ & $\mathrm{NL}$ & $34 / M$ & 38 & 89 & 342 & 18 \\
\hline GDD & $\mathrm{Sz}$ & Slow & $\begin{array}{l}\text { Volume } \\
\text { loss, PVL }\end{array}$ & $21 / F$ & 38 & 187 & 500 & 53 \\
\hline GDD & DD & ND & Thin CC & $22 / \mathrm{M}$ & 40 & 321 & 575 & 56 \\
\hline GDD & NS & $\begin{array}{l}\text { Multifocal } \\
\text { spikes }\end{array}$ & Volume loss & 29/M & 40 & 244 & 622 & 21 \\
\hline $\begin{array}{l}\text { Refractory } \\
\text { seizures }\end{array}$ & NS & $\begin{array}{l}\text { Multifocal } \\
\text { spikes }\end{array}$ & $\mathrm{NL}$ & $6 / F$ & 40 & 336 & 602 & 48 \\
\hline GDD & ADHD & Focal Spikes & DWMA & $22 / \mathrm{M}$ & 45 & 201 & 619 & 18 \\
\hline
\end{tabular}

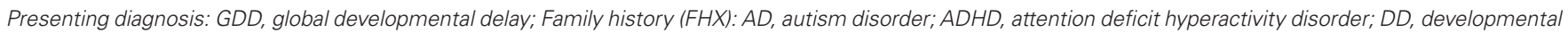

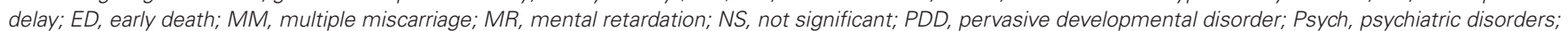

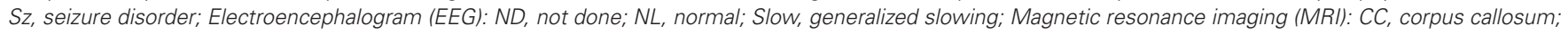

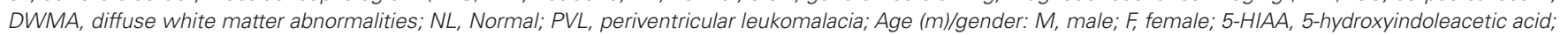
HVA, homovanillic acid; 3-OMD, 3-O-methyldopa. 
group was significantly older $($ mean $=66.0$ months, $\mathrm{SE}=15.10)$ than the high $\mathrm{BH}_{4}$ group $[$ mean $=24.6, \mathrm{SE}=4.3 ; t(20)=2.66$, $p<0.05]$ and age was found to correlate negatively with cerebrospinal fluid $\mathrm{BH}_{4}$ level $[r(19)=-0.44, p<0.05]$. There was a significantly greater percentage of patients in the high $\mathrm{BH}_{4}$ group $(60 \%)$ with discharges on electroencephalogram as compared to the low $\mathrm{BH}_{4}$ group $\left[9 \%, \chi^{2}(1)=4.75, p<0.05\right]$. The two groups were not different in the proportion of patients with a positive family history, slowing on electroencephalogram or abnormal magnetic resonance imaging findings. 5-Hydroxyindoleacetic acid, homovanillic acid and 3-O-methyldopa were not significantly different between groups. The majority of the children with autism were in the low $\mathrm{BH}_{4}$ group $(80 \%)$ as compared to the high $\mathrm{BH}_{4}$ group. Defining these groups also enabled us to assign prior probabilities of 0.70 for the low group and 0.30 for the high group.

\section{SERUM CITRULLINE AND METHIONINE CONCENTRATIONS DIFFERENTIATE THE HIGH AND LOW BH GROUPS}

As discussed above, amino acids believed to be related to $\mathrm{BH}_{4}$ metabolism were entered into a discriminant analysis to determine if concentrations of specific amino acids could differentiate individuals in the high and low $\mathrm{BH}_{4}$ groups. The amino acids, citrulline and methionine, significantly contributed to the discriminant function [Wilks' lambda $F(2,8)=6.66, p<0.05$ ]. As can be seen in Figure 1A, citrulline was significantly elevated in the low $\mathrm{BH}_{4}$ group $[t(10)=2.34, p<0.05]$ while methionine was non-significantly depressed in the low $\mathrm{BH}_{4}$ group $[t(10)=1.32, p>0.05]$. Assuming equal prior group probabilities, the discriminant function did not make any errors, resulting in perfect sensitivity, specificity, and positive and negative predictive values (Table 2). The scatter plot of all citrulline-to-methionine values, along with the criteria that divided the two groups assuming an equal prior group probability, is displayed in Figure 1B. The discriminant function produced using the observed prior group probabilities misclassified one patient from the high $\mathrm{BH}_{4}$ group but none of the patients from the low $\mathrm{BH}_{4}$ group, resulting in a perfect sensitivity and negative predictive value. Since we found an age difference between the two $\mathrm{BH}_{4}$ groups, we added age and interactions of age with amino acid markers into the initial stepwise discriminant analysis. This analysis selected citrulline and the interaction between citrulline and age [Wilks' lambda $F(2,8)=8.69, p<0.01$ ]. Assuming equal prior group probabilities, the discriminant function classified one
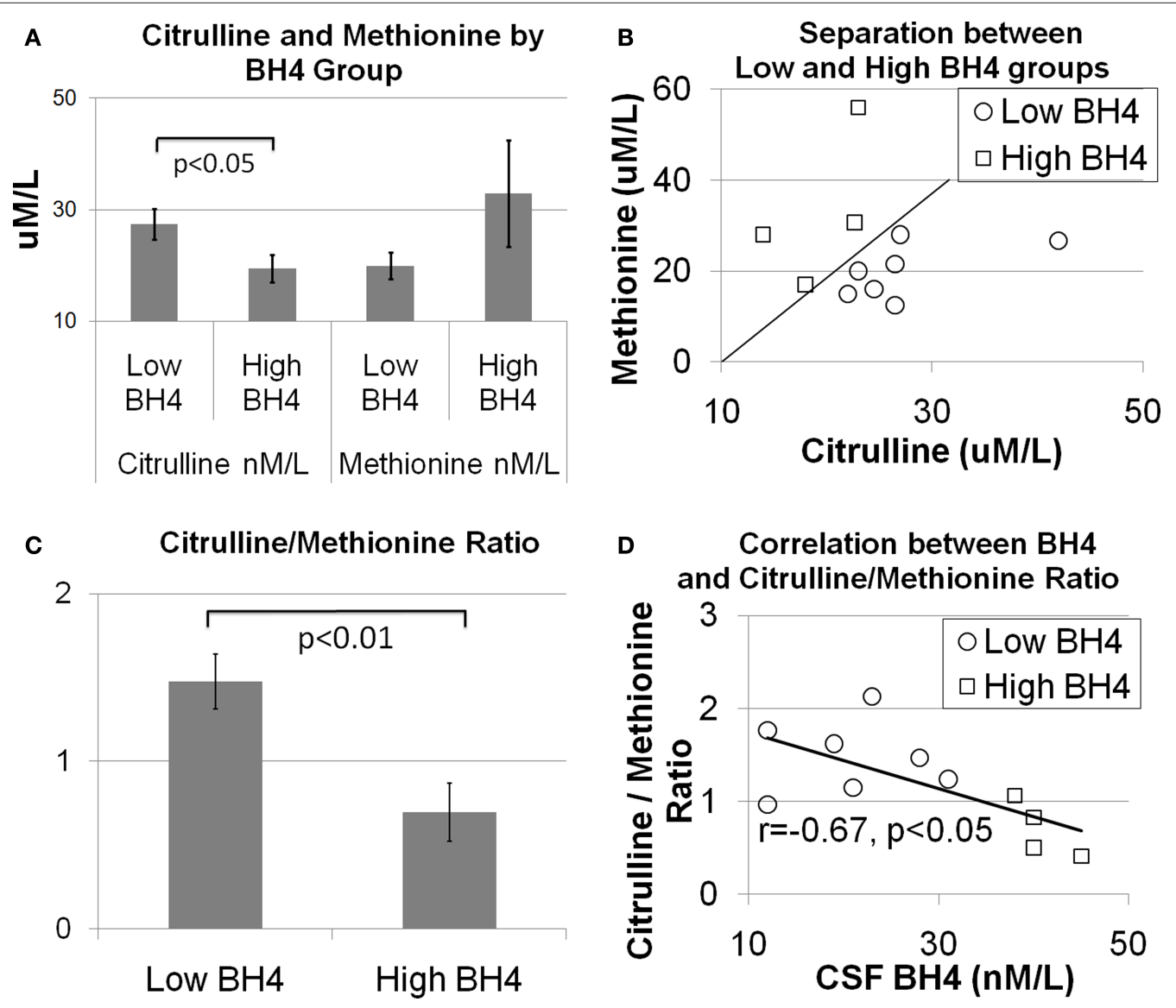

FIGURE 1 |The relationship between central tetrahydrobiopterin $\left(\mathrm{BH}_{4}\right)$ concentration and serum citrulline and methionine concentration.

(A) Citrulline was found to be higher and methionine was found to be lower in individuals in the low $\mathrm{BH}_{4}$ group as compared to individuals in the high $\mathrm{BH}_{4}$ group.

(B) Scatter plot of citrulline and methionine values illustrating the separation between the low and high $\mathrm{BH}_{4}$ groups. The diagonal line illustrates the group separate. (C) The ratio of citrulline-to-methionine is significantly different between the low and high $\mathrm{BH}_{4}$ groups and (D) correlated with the central $\mathrm{BH}_{4}$ concentration. 
Table 2 | Performance statistics for discriminant functions.

\begin{tabular}{llcrrr}
\hline Model variables & Prior group & Sensitivity (\%) & Specificity (\%) & \multicolumn{1}{c}{$\begin{array}{l}\text { Positive predictive } \\
\text { value (\%) }\end{array}$} & $\begin{array}{l}\text { Negative predictive } \\
\text { value (\%) }\end{array}$ \\
& probability & & & 100.00 & 100.00 \\
Citrulline and methionine & Equal & 100.00 & 100.00 & 75.00 & 100.00 \\
Citrulline and methionine & Observed & 100.00 & 100.00 & 100.00 & 80.00 \\
Citrulline and citrulline $\times$ age & Equal & 85.71 & 100.00 & 100.00 & 100.00 \\
Citrulline and citrulline $\times$ age & Observed & 100.00 & 100.00 & 100.00 & 80.00 \\
Citrulline/methionine ratio & Equal & 85.71 & 75.00 & 87.50 & 100.00 \\
Citrulline/methionine ratio & Observed & 100.00 & & & \\
\hline
\end{tabular}

observation in the low $\mathrm{BH}_{4}$ group incorrectly resulting in high sensitivity, specificity, and positive and negative predictive values. Assuming the observed prior group probabilities, the discriminant function classified all observations correctly, resulting in perfect sensitivity, specificity, and positive and negative predictive values.

\section{THE CITRULLINE-TO-METHIONINE RATIO: A SIMPLE INDEX THAT DIFFERENTIATES HIGH AND LOW $\mathrm{BH}_{4}$ GROUPS}

To produce a simple, easy to calculate index of cerebrospinal fluid $\mathrm{BH}_{4}$, the ratio of citrulline-to-methionine was calculated. Figure $1 \mathrm{C}$ demonstrates that the citrulline-to-methionine ratio was significantly higher in the low $\mathrm{BH}_{4}$ group as compared to the high $\mathrm{BH}_{4}$ group $[t(10)=3.44, p<0.01]$. In addition, the citrulline-to-methionine ratio was found to significantly correlate with cerebrospinal fluid $\mathrm{BH}_{4}$ levels $[r(9)=-0.67, p<0.05$; see Figure 1D]. The discriminant function with the citrulline-to-methionine ratio was statistically significantly [Wilks' lambda $F(1,9)=11.35, p<0.01$ ] The discriminant function with equal prior group probabilities classified one observation in the low $\mathrm{BH}_{4}$ group incorrectly but no observations in the high $\mathrm{BH}_{4}$ group incorrectly, resulting in a perfect specificity and positive predictive value but a lower sensitivity and negative predictive value. The discriminant function produced using the observed prior group probabilities misclassified one observation in the high $\mathrm{BH}_{4}$ group and no patients in the low $\mathrm{BH}_{4}$ group, resulting in a perfect sensitivity and negative predictive value. Including age and the interaction of age with the citrullineto-methionine ratio as predictors in the stepwise analysis did not improve the model.

\section{A PRACTICAL METHOD FOR APPLYING THE DISCRIMINANT FUNCTION RESULTS}

In order to make the discriminant functions clinically useful, a simple method for calculating group membership is discussed. Since the discriminant function that included methionine and citrulline did not make assumptions about prior group probabilities and provided perfect discrimination between groups, it was used to calculate group membership. Group membership can be calculated by using Eq. 1 with a patient's methionine and citrulline values. If the resulting quantity is greater than 0 , then the patient would be considered to be in the low $\mathrm{BH}_{4}$ group. If the quantity resulting from Eq. 1 is less than 0, then the patient would be classified in the high $\mathrm{BH}_{4}$ group.

$$
\begin{aligned}
\text { Group } & =-3.48+0.39 \times \text { citrulline }(\mu \mathrm{M} / \mathrm{L}) \\
& -0.22 \times \text { methionine }(\mu \mathrm{M} / \mathrm{L}) .
\end{aligned}
$$

Alternatively, the citrulline-to-methionine ratio could be used with the caveat that the value does not appear to discriminate among groups as well as the quantity derived from Eq. 1. The dividing point for this ratio, as calculated by the discriminant function, is 1.05 assuming equal group probabilities and 0.98 assuming observed group probabilities.

\section{DISCUSSION}

This report examined cerebrospinal fluid $\mathrm{BH}_{4}$ concentrations in 20 children and adolescents with neurological disorders. First, cluster analysis was used to determine if, and how many, $\mathrm{BH}_{4}$ groups were in the patient population. Two groups of patients were found: $70 \%$ of the patients had cerebrospinal fluid $\mathrm{BH}_{4}$ concentrations of $31 \mathrm{nM} / \mathrm{L}$ and below and $30 \%$ had concentrations of $35 \mathrm{nM} / \mathrm{L}$ and above. Significantly more patients in the group with higher $\mathrm{BH}_{4}$ concentrations demonstrated epileptiform activity on electroencephalogram, and the majority of the children with the diagnosis of autism were in the low $\mathrm{BH}_{4}$ group. The discriminant analysis revealed that the serum concentration of two amino acids, citrulline and methionine, could discriminate the high and low $\mathrm{BH}_{4}$ groups and that the ratio of citrulline-to-methionine correlated with the cerebrospinal fluid $\mathrm{BH}_{4}$ concentration. The relevance of these specific amino acids to $\mathrm{BH}_{4}$ metabolism is discussed below. The relation identified in the analysis above is significant in that it implies that measurement of these peripheral markers of metabolism may be useful for identifying individuals with low central $\mathrm{BH}_{4}$ levels who might benefit from $\mathrm{BH}_{4}$ supplementation.

The low and high $\mathrm{BH}_{4}$ groups appear to correspond to patients with low-to-normal and normal-to-high cerebrospinal fluid $\mathrm{BH}_{4}$ concentrations, respectively. It is somewhat surprising that a group with markedly low cerebrospinal fluid $\mathrm{BH}_{4}$ levels was not found since some research suggests that cerebrospinal fluid $\mathrm{BH}_{4}$ concentrations in autism might be depressed; however, only a subset of individuals within our population had features of autism. Two studies that measured $\mathrm{BH}_{4}$ concentrations in children with autism have found concentrations, for the most part, below the lowest cerebrospinal fluid $\mathrm{BH}_{4}$ concentration of our patient population (Tani et al., 1994; Fernell et al., 1997). However, another study found cerebrospinal fluid $\mathrm{BH}_{4}$ concentrations in a group of autistic children more comparable with our findings (Danfors et al., 2005). In addition, Danfors et al. (2005) also excluded about one-third of the children they evaluated due to cerebrospinal fluid $\mathrm{BH}_{4}$ concentration greater than $30 \mu \mathrm{mol} / \mathrm{mL}$, a proportion very close to the proportion of patients in the high $\mathrm{BH}_{4}$ group reported in this study. The great majority of studies that have shown an improvement 
in autism symptomatology with $\mathrm{BH}_{4}$ supplementation did not measure blood, urine or cerebrospinal fluid $\mathrm{BH}_{4}$ concentrations. Thus, it is difficult to know whether $\mathrm{BH}_{4}$ supplementation affected children with low, normal or high $\mathrm{BH}_{4}$ levels or children with a mixture of these levels. In fact it may be better to think of patient populations that might have over activation of metabolic systems associated with $\mathrm{BH}_{4}$ utilization, such as autism, as having a relative insufficiency in $\mathrm{BH}_{4}$, rather than a $\mathrm{BH}_{4}$ deficiency. In such a case, the central $\mathrm{BH}_{4}$ concentration could be low normal and still be insufficient to meet the metabolic demands of the individual.

One of the important insights that this report highlights, regardless of the exact cerebrospinal fluid $\mathrm{BH}_{4}$ concentrations of our patients, is that central nervous system $\mathrm{BH}_{4}$ levels may very well be associated with peripheral metabolic pathways. As discussed above, we believe that this relationship is a result of consumption and recycling of $\mathrm{BH}_{4}$ by non-central metabolic pathways. Additionally, polymorphisms in one of the several genes that code for $\mathrm{BH}_{4}$ production could be associated with reduced or inefficient $\mathrm{BH}_{4}$ production or recycling. Such a notion would be consistent with the significant nominal association between the 6-pyruvoyl-tetrahydropterin synthase gene and autism (Schnetz-Boutaud et al., 2009). Of course, the population studied does not represent typically developing individuals, and many of the children we examined may be expected to have activation of metabolic pathways that consume $\mathrm{BH}_{4}$. This limits the application of the relationship observed to those children and adolescents with neurodevelopmental disorders. However, this population may be one of the most important populations to which to apply the findings of this study since they may be high consumers of $\mathrm{BH}_{4}$ and have a high need for $\mathrm{BH}_{4}$ supplementation. Indeed, several studies have demonstrated improvement in development and behavior with supplementation of $\mathrm{BH}_{4}$ in children with autism (Naruse et al., 1984, 1990a,b; Nagahata et al., 1990; Nakane et al., 1990; Takesada et al., 1992; Fernell et al., 1997; Danfors et al., 2005; Frye et al., 2010).

Children with autism have symptoms consistent with a central $\mathrm{BH}_{4}$ deficiency. For example, $\mathrm{BH}_{4}$ deficiency can result in low production of monoamine neurotransmitters, including serotonin, dopamine, and norepinephrine. There is a significant amount of evidence that deficits in these monoamine neurotransmitters are present in some children with autism. Dysfunction in the serotonin system in autism has been documented by several investigators (Pardo and Eberhart, 2007). Children with autism have clinical symptoms, such as obsessive-compulsive disorder and anxiety, seen in other disorders where serotonin deficiency has been implicated (McDougle et al., 1995), and there are many studies that suggest that selective-serotonin reuptake inhibitors may be efficacious in the treatment of autistic symptoms (Kolevzon et al., 2006; Posey et al., 2006a). Children with autism have a high incidence of sleep initiation and maintenance disorders, suggesting a deficit in melatonin, a metabolite of serotonin (Richdale and Schreck, 2009). Children with autism have a high rate of executive function, attention regulation and hyperactivity, all symptoms suggesting dopamine and norepinephrine deficits (Bramham et al., 2009; Corbett et al., 2009; Jahromi et al., 2009). Such behaviors are mitigated by psychopharmaceuticals designed to increase levels of dopamine and norepinephrine in children with autism (Posey et al., 2006b; Troost et al., 2006). In addition, positron emission tomography has demonstrated a reduction in the baseline increase in dopamine $\mathrm{D}_{2}$ receptor binding after 12 weeks of $\mathrm{BH}_{4}$ supplementation (Fernell et al., 1997). It is important to state that it is highly likely that not all children with autism have low monoamine neurotransmitters, as the support for this idea across studies in mixed (Kolevzon et al., 2006; Posey et al., 2006b; Soorya et al., 2008). This is most likely the result of the heterogeneity in the biological mechanisms underlying autism and supports the need to develop biochemical markers of central metabolic processes in children with autism and other neurodevelopmental disorders, and might explain why not all children with autism respond to $\mathrm{BH}_{4}$ supplementation.

Low central nervous system $\mathrm{BH}_{4}$ levels can result in several secondary central nervous system consequences. First, $\mathrm{BH}_{4}$ is necessary for the production of nitric oxide, a soluble signaling molecule that appears to be important for cell proliferation, neuronal motility, and synaptic maturation during development (Tegenge and Bicker, 2009) and communication between neurons and both neuronal and non-neuronal cells (Garthwaite, 2008). Second, $\mathrm{BH}_{4}$ is associated with growth factors, including nerve growth factors, in animal models (Anastasiadis et al., 1997). Third, $\mathrm{BH}_{4}$ has been shown to be a protective factor for nitric oxide toxicity and a superoxide radical scavenger (Kojima et al., 1995; Koshimura et al., 1998). Fourth, reduced levels of monoamine neurotransmitters could result in dysfunction of important neural pathways, leading to underdevelopment of such important pathways. Fifth, $\mathrm{BH}_{4}$ is an enhancer of the synaptic release of a wide range of neurotransmitters including the catecholamines, serotonin, acetylcholine, glutamate, and gamma aminobutyric acid (Koshimura et al., 1990; Mataga et al., 1992). Thus, a reduction in $\mathrm{BH}_{4}$ could result in a reduction in neurotransmitter release during a critical time in development. Clearly $\mathrm{BH}_{4}$ is involved in several metabolic and neurotransmitter pathways critical for brain function, and development and low $\mathrm{BH}_{4}$ levels during development could have devastating consequences on brain development.

Two serum amino acids, citrulline and methionine, were found to differentiate high and low cerebrospinal fluid $\mathrm{BH}_{4}$ concentration in individuals with neurological disorders. In addition, the ratio of these two amino acids was correlated with the cerebrospinal fluid $\mathrm{BH}_{4}$ concentration. Since these amino acids are an integral part of metabolic pathway that uses $\mathrm{BH}_{4}$ both systemically and centrally, it is suggested that dysfunction of such metabolic pathways is linked to changes in $\mathrm{BH}_{4}$ levels. Specifically, a reduction in serum methionine is proposed to be linked to a reduction in folate availability and activation of the oxidative stress pathway, and an increase in serum citrulline is proposed to be linked to an increase in nitric oxide production from overactive inflammatory pathways. This notion is supported by the fact that some children with autism demonstrate these metabolic abnormalities and respond to $\mathrm{BH}_{4}$ supplementation.

Several aspects of the hypothesized relation between cerebrospinal fluid $\mathrm{BH}_{4}$ concentration and serum amino acids were not verified by our analysis. For example, arginine was not found to be reduced. This is most likely due to the fact that changes in metabolism, including $\mathrm{BH}_{4}$ metabolism, are due to long-standing chronic effects of disease. In such states, enzymes are regulated to compensate for the abnormal metabolic state. For example, down regulation 
of arginase, the enzyme that catalyzes the breakdown of arginine to urea and ornithine in the urea cycle results in an increase in citrulline (Rabier and Kamoun, 1995; Tenu et al., 1999). Inhibition of arginase would prevent the decrease in arginine and would result in an increase in citrulline as seen in this study.

Clearly, future studies will need to examine other biomarkers of oxidative stress, nitric oxide, and inflammation to understand whether they correlate with the serum amino acid markers identified in this study and systemic and/or cerebrospinal fluid $\mathrm{BH}_{4}$

\section{REFERENCES}

Anastasiadis, P.Z., Bezin,L.,Imerman,B.A., Kuhn, D. M., Louie, M. C., and Levine, R. A. (1997). Tetrahydrobiopterin as a mediator of PC12 cell proliferation induced by EGF and NGF. Eur. J. Neurosci. 9, 1831-1837.

Bramham, J., Ambery, F., Young, S., Morris, R., Russell, A., Xenitidis, K., Asherson, P., and Murphy, D. (2009). Executive functioning differences between adults with attention deficit hyperactivity disorder and autistic spectrum disorder in initiation, planning and strategy formation. Autism 13, 245-264.

Castellani, M. L., Conti, C. M., Kempuraj, D. J., Salini, V., Vecchiet, J., Tete, S., Ciampoli, C., Conti, F., Cerulli, G., Caraffa, A., Antinolfi, P., Galzio, R., Shaik, Y., Theoharides, T. C., De Amicis, D., Perrella, A., Cuccurullo, C., Boscolo, P., Felaco, M., Doyle, R., Verrocchio, C., and Fulcheri, M. (2009). Autism and immunity: revisited study. Int. J. Immunopathol. Pharmacol. 22, 15-19.

Corbett, B.A., Constantine, L. J., Hendren, R., Rocke, D., and Ozonoff, S. (2009). Examining executive functioning in children with autism spectrum disorder, attention deficit hyperactivity disorder and typical development. Psychiatry Res. 166, 210-222.

Danfors, T., von Knorring, A. L., Hartvig, P., Langstrom, B., Moulder, R., Stromberg, B., Torstenson, R., Wester, U., Watanabe, Y., and Eeg-Olofsson, O. (2005). Tetrahydrobiopterin in the treatment of children with autistic disorder: a double-blind placebocontrolled crossover study. J. Clin. Psychopharmacol. 25, 485-489.

Deth, R., Muratore, C., Benzecry, J., Power-Charnitsky, V. A., and Waly, M. (2008). How environmental and genetic factors combine to cause autism: a redox/methylation hypothesis. Neurotoxicology 29, 190-201.

Dietert, R. R., and Dietert, J. M. (2008). Potential for early-life immune insult including developmental immunotoxicity in autism and autism spectrum disorders: focus on critical windows of immunevulnerability.J. Toxicol.Environ. Health B Crit. Rev. 11, 660-680.
Fernell,E., Watanabe, Y., Adolfsson, I., Tani, Y., Bergstrom, M., Hartvig, P., Lilja, A., von Knorring, A. L., Gillberg, C., and Langstrom, B. (1997). Possible effects of tetrahydrobiopterin treatment in six children with autism - clinical and positron emission tomography data: a pilot study. Dev. Med. Child. Neurol. 39, 313-318.

Frye, R. E., Huffman, L. C., and Elliot, G. R. (2010). Tetrahydrobiopterin as a novel therapeutic intervention for autism. Neurotherapeutics (in press).

Garthwaite, J. (2008). Concepts of neural nitric oxide-mediated transmission. Eur. J. Neurosci. 27, 2783-2802.

Howells, D., and Hyland, K. (1987). Direct analysis of tetrahydrobiopterin in cerebrospinal fluid by high-performance liquid chromatography with redox electrochemistry: prevention of autoxidation during storage and analysis. Clin. Chim. Acta 167, 23-30.

Hyland, K., Surtees, R. A., Heales, S. J., Bowron, A., Howells, D. W., and Smith, I. (1993). Cerebrospinal fluid concentrations of pterins and metabolites of serotonin and dopamine in a pediatric reference population. Pediatr. Res. 34, 10-14.

Jahromi, L. B., Kasari, C. L., McCracken, J. T., Lee, L. S., Aman, M. G., McDougle, C. J., Scahill, L., Tierney, E., Arnold, L. E., Vitiello, B., Ritz, L., Witwer, A., Kustan, E., Ghuman, J., and Posey, D. J. (2009). Positive effects of methylphenidate on social communication and self-regulation in children with pervasive developmental disorders and hyperactivity. J. Autism Dev. Disord. 39, 395-404.

James, S. J., Cutler, P., Melnyk, S., Jernigan, S., Janak, L., Gaylor, D. W., and Neubrander, J. A. (2004). Metabolic biomarkers of increased oxidative stress and impaired methylation capacity in children with autism. Am. J. Clin. Nutr. 80, 1611-1617.

Kern, J. K., and Jones, A. M. (2006). Evidence of toxicity, oxidative stress, and neuronal insult in autism. J. Toxicol. Environ. Health B Crit. Rev. 9, 485-499.

Kojima, S., Ona, S., Iizuka, I., Arai, T., Mori, H., and Kubota, K. (1995). Antioxidative activity of 5,6,7,8-

concentration. Such information will further our understanding of the biological basis of autism and help validate the ratio of citrulline-to-methionine as a biomarker for central $\mathrm{BH}_{4}$ concentration in other disorders.

\section{ACKNOWLEDGMENTS}

This study was supported by NS046565 to Dr. Richard Frye. The author would like to thank Laura deSouza and Christine Younan for their technical help with this study.

tetrahydrobiopterin and its inhibitory effect on paraquat-induced cell toxicity in cultured rat hepatocytes. Free Radic. Res. 23, 419-430.

Kolevzon, A., Mathewson, K. A., and Hollander, E. (2006). Selective serotonin reuptake inhibitors in autism: a review of efficacy and tolerability. $J$. Clin. Psychiatry 67, 407-414.

Koshimura, K., Miwa, S., Lee, K., Fujiwara, M., and Watanabe, Y. (1990). Enhancement of dopamine release in vivo from the rat striatum by dialytic perfusion of 6R-L-erythro-5,6,7,8tetrahydrobiopterin. J. Neurochem. 54, 1391-1397.

Koshimura, K., Murakami, Y., Tanaka, J., and Kato, Y. (1998). Self-protection of PC1 2 cells by $6 \mathrm{R}$-tetrahydrobiopterin from nitric oxide toxicity. J. Neurosci. Res. 54, 664-672.

Mataga, N., Imamura, K., and Watanabe, Y. (1992). L-threo-3,4dihydroxyphenylserine enhanced ocular dominance plasticity in adult cats. Neurosci. Lett. 142, 115-118.

McDougle, C. J., Kresch, L. E., Goodman, W. K., Naylor, S. T., Volkmar, F. R., Cohen, D. J., and Price, L. H. (1995). A case-controlled study of repetitive thoughts and behavior in adults with autistic disorder and obsessivecompulsive disorder. Am. J. Psychiatry 152, 772-777.

Nagahata, M., Kazamatsuri, H., Naruse, H., Yamazaki, K., Takesada, M., Nakane, Y., Kaihara, S., and Ohashi, T. (1990). Clinical evaluation of aproterin hydrochloride (R-THBP. SUN 0588) on infantile autism - a multicenter cooperative study. Rinsho Iyaku 6, 1877-1899 (in Japanese).

Nakane, Y., Asuo, T., Shimogawa, S., Fujiwara, T., Kawabata, Y., and Kubota, J. (1990). Clinical efficacy and effects on physical development of long-term treatment of R-tetrahydrobiopterin (R-THBP,SUN 0588) for autism. Kiso To Rinsho 24, 4579-4598 (in Japanese).

Naruse, H., Hayashi, T., Takesada, M. (1984). "A preliminary study on clinical effect of tetrahydrobiopterin in infantile autism (in Japanese)," in Reports in 1983 for New Drug Development (Tokyo: Ministry of Health and Welfare), 71-81.
Naruse, H., Takesada, M., Nagahata, M., Kazamatsuri, H., Nakane, Y., and Yamazaki, K. (1990b). An open clinical study of apropterin hydrochloride (R-tetrahydrobiopterin SUN 0588) in infantile autism - clinical study using a Rating Scale for Abnormal Behaviors in Children. Rinsho Iyaku 6, 1859-1875 (in Japanese).

Naruse, H., Takesada, M., Nakane, Y., Yamazaki, K., Uchiyama, T., Kaihara, S., and Ohashi, T. (1990a). Clinical evaluation of R-tetrahydrobiopterin (SUN 0588) on infantile autism - a double-blind comparative study using placebo as a control. Rinsho Iyaku 6, 1343-1368 (in Japanese).

Pardo, C. A., and Eberhart, C. G. (2007). The neurobiology of autism. Brain Pathol. 17, 434-447.

Pardo, C.A., Vargas, D.L., andZimmerman, A.W.(2005).Immunity, neuroglia and neuroinflammation in autism. Int. Rev. Psychiatry 17, 485-495.

Posey, D. J., Wiegand, R. E., Wilkerson, J., Maynard, M., Stigler, K. A., and McDougle, C. J. (2006a). Open-label atomoxetine for attention-deficit/ hyperactivity disorder symptoms associated with high-functioning pervasive developmental disorders. J. Child Adolesc. Psychopharmacol. 16, 599-610.

Posey, D. J., Erickson, C. A., Stigler, K. A., and McDougle, C. J. (2006b). The use of selective serotonin reuptake inhibitors in autism and related disorders. J. Child Adolesc. Psychopharmacol. 16, 181-186.

Rabier, D., and Kamoun, P. (1995). Metabolism of citrulline in man. Amino Acids 9, 299-316.

Richdale, A. L., and Schreck, K. A. (2009). Sleep problems in autism spectrum disorders: prevalence, nature, and possible biopsychosocial aetiologies. Sleep Med. Rev. 13, 403-411.

Schnetz-Boutaud, N. C., Anderson, B. M., Brown, K.D., Wright,H.H.,Abramson, R. K., Cuccaro, M. L., Gilbert, J. R., Pericak-Vance, M. A., and Haines, J. L. (2009). Examination of tetrahydrobiopterin pathway genes in autism. Genes Brain Behav. 8, 753-757.

Soorya, L., Kiarashi, J., and Hollander, E. (2008). Psychopharmacologic 
interventions for repetitive behaviors in autism spectrum disorders. Child Adolesc. Psychiatr. Clin. N. Am. 17, 753-771.

Takesada, M., Naruse, H., and Nagahata, M. (1992). "An open clinical study of sapropterin hydrochloride (R-tetrahydrobiopterin, R-THBP) in infantile autism: clinical effects and long-term follow-up," in Neurobiology of Infantile Autism: Proceedings of the International Symposium on Neurobiology of Infantile Autism, Tokyo, 10-11 November 1990, eds H. Naruse and E. Ornitz (New York: Elsevier Science Publishers), 355-358.

Tani, Y., Fernell, E., Watanabe, Y., Kanai, T., and Langstrom, B. (1994). Decrease in $6 \mathrm{R}-5,6,7,8$-tetrahydrobiopterin content in cerebrospinal fluid of autistic patients. Neurosci. Lett. 181, 169-172.

Tegenge, M.A., and Bicker, G. (2009). Nitric oxide and cyclic GMP signal transduction positively regulates the motility of human neuronal precursor (NT2) cells. J. Neurochem. 110, 1828-1841.

Tenu, J. P., Lepoivre, M., Moali, C., Brollo, M., Mansuy, D., and Boucher, J. L. (1999). Effects of the new arginase inhibitor N(omega)-hydroxy-nor-Larginine on NO synthase activity in murine macrophages. Nitric Oxide 3, 427-438.

Thony, B., Auerbach, G., and Blau, N. (2000). Tetrahydrobiopterin biosynthesis, regeneration and functions. Biochem. J. 347, 1-16.

Troost, P. W., Steenhuis, M. P., TuynmanQua, H. G., Kalverdijk, L. J., Buitelaar,
J. K., Minderaa, R. B., and Hoekstra, P. J. (2006). Atomoxetine for attention-deficit/hyperactivity disorder symptoms in children with pervasive developmental disorders: a pilot study. J. Child Adolesc. Psychopharmacol. 16, 611-619.

Willoughby, R. E., Opladen, T., Maier, T., Rhead, W., Schmiedel, S., Hoyer, J., Drosten, C., Rupprecht, C. E., Hyland, K., and Hoffmann, G. F. (2009). Tetrahydrobiopterin deficiency in human rabies. J. Inherit. Metab. Dis. $32,65-72$.

Conflict of Interest Statement: Dr Richard Frye has received honoraria from BioMarin, the manufacture of Kuvan (a formulation of tetrahydrobiopterin), for speaking at a workshop on tetrahydrobiopterin in neurodevelopmental disorders.

Received: 10 March 2010; paper pending published: 30 March 2010; accepted: 22 June 2010; published online: 08 July 2010. This article was submitted to Frontiers in Neuropharmacology, a specialty of Frontiers in Neuroscience.

Citation: Frye RE (2010) Central tetrahydrobiopterin concentration in neurodevelopmental disorders. Front. Neuropharma. 4:52. doi: 10.3389/fnins.2010.00052

Copyright (c) 2010 Frye. This is an openaccess article subject to an exclusive license agreement between the authors and the Frontiers Research Foundation, which permits unrestricted use, distribution, and reproduction in any medium, provided the original authors and source are credited. 